\title{
Correlation technique using of-the-shelf CW DFB diode laser for interrogation of fiber optic sensors
}

\author{
M.G. Shlyagin ${ }^{a, *}$, L. A. Arias ${ }^{a, b}$, and Jorge H. López ${ }^{a, c}$ \\ ${ }^{a}$ Departamento de Óptica, Centro de Investigación Científica y de Educación Superior de Ensenada, \\ Carretera Tijuana-Ensenada 3918, Baja California, 22860, México. \\ *e-mail:mish@cicese.mx \\ ${ }^{b}$ Universidad Tecnológica del Valle de Toluca, Santa María Atarasquillo, 52044, México. \\ ${ }^{c}$ Centro de Investigaciones en Óptica, Aguascalientes, 20200, México.
}

Received 13 December 2020; accepted 29 December 2020

\begin{abstract}
The paper presents a simple fiber-optic sensor technique based on methods of correlation optical time domain reflectometry. A correlation reflectometry technique can measure a distribution of the reflection coefficient along the optical fiber by calculating the correlation function between a probe optical signal (reference) and the signal returned back due to reflections or/and back-scattering from the fiber under the test. To obtain the best sensor performance, the probe signal power should be a truly random function of time. As an optical source, we use a free-running DFB laser diode operating in a continuous wave regime without any external modulation. To generate the probe test signal, laser light is passed through an interferometer with an optical path difference much longer than the coherence length of the laser light. The light intensity at the interferometer output has a truly random fluctuations, and its autocorrelation function is suitable for correlation optical reflectometry. We present results of experimental verification of the techniques in different sensor configurations. Multipoint sensor using very low reflective fiber Bragg gratings with reflectivity of $0.05 \%$ printed in a long SMF-28 optical fiber was demonstrated.
\end{abstract}

Keywords: Fiber optic sensors; fiber Bragg gratings; multiplexing.

PACS: 42.55.Px; 42.81.-i; 42.81.Pa; 07.60.Vg

\section{Introduction}

The optical fibers can be used as sensors to measure mechanical stress, deformation, temperature, pressure, and other parameters. The small size and the fact that electricity does not circulate through them, give certain advantages over electrical sensors. They are immune to electromagnetic fields, in addition to the absence of electrical connections. Such properties of optical fibers allow them to be used in a highrisk explosive environment (e.g. mines, pipelines). One of the main advantages of fiber optic sensors is the capability to perform distributed sensing. There are several types of distributed fiber optic sensors using light scattering effects such as Rayleigh, Raman, or Brillouin backscattering, as well as arrays of lumped weak reflectors [1-3]. Sensors based on in-fiber Bragg gratings (FBGs) have demonstrated a great multiplexing capacity which allows having a large number of sensors in a single optical fiber and to build quasidistributed sensors with lengths of up to several kilometers. The wavelength-division multiplexing (WDM) method and Time-division multiplexing (TDM) dominate application of FBG sensing [3-6].

The ability to multiplex many sensors is important for practical reasons since it can reduce complexity and cost per sensing point. Therefore, the interrogation techniques which can be easily integrated with the multiplexing concept can be of practical interest.

Interrogation methods based on the Optical Time Domain Multiplexing technique require the use of nominally identical
FBGs with the same Bragg wavelength. As a result, FBGs with very low reflectivity have to be used in order to reduce a cross-talk noise level [5]. For usual pulse OTDR, that results in problems associated with the need to use short high energy pulses and long averaging time in order to obtain a good signal-to-noise ratio. To overcome this problem, the optical energy emitted in a single short impulse can be spread over a much longer time interval and the correlation detection of signals returned from the sensing fiber can be used. For proper measuring of distribution of reflectors along the optical fiber, the autocorrelation function of the probe light has to be like the delta-function. Correlation signal processing is widely used in different measuring techniques for the detection and recognition of weak signals in a presence of a high level of noise. In optics, it is used in correlation OTDRs, LIDARs $[7,8]$, etc.

For optical time domain reflectometers multiplexed sensors so -called Code Division Multiplexing has been demonstrated [7]. It is based on the correlation property of a pseudorandom binary sequence code. Usually, a laser light intensity is modulated with a pseudorandom bit sequence, and demultiplexing of signals from different reflectors can be performed by calculating the correlation function using a different delayed version of the code.

In this paper, we demonstrate a new optical reflectometer based on correlation detection [9-11]. Unlike most reported techniques, the proposed technique does not need any shortpulse sources or light intensity modulators but uses an unmodulated CW DFB laser whose natural phase noise is con- 


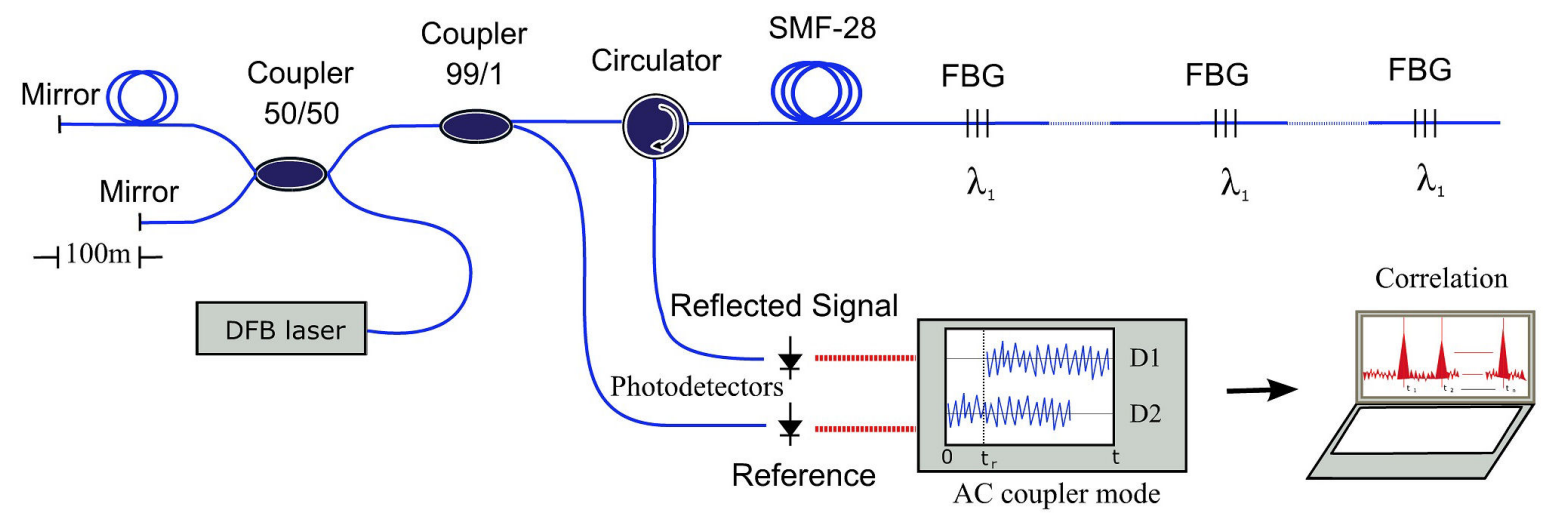

FIGURE 1. Experimental set up.

verted into random amplitude modulation by an unbalanced interferometer. The distribution of the back-reflected light is obtained by calculating the cross-correlation between the reference and back-reflected optical signals. We describe the operating principle of the proposed technique and present the results of the experimental verification.

\section{Principle of operation and experiment}

Figure 1 shows the schematic diagram for experimental verification of the proposed technique. As a light source, we used a conventional CW DFB diode laser with a simple current/temperature controller. Modulation of the laser light intensity is not required in our scheme. However, if necessary, the wavelength of the output light can be slowly swept within the interval of 2 nanometers by sweeping the current of the Peltier element and thus changing the temperature of the laser diode crystal. The sweep of wavelength was used for measur- ing the reflection spectra of FBGs. The laser output was coupled to an imbalanced interferometer with a length difference between arms of 100 meters; that is longer than the coherence length of the laser light. At the interferometer output, two waves are delayed much more than the coherence time of the laser light. Therefore, the mean light power at interferometer output is stable and no interference fringes can be observed. However, an instant light power fluctuates rapidly due to the efficient conversion of the input phase noise into intensity noise. Figure 2 shows an example of a realization of the reference signal from the photodetector with a bandwidth of $10 \mathrm{MHz}$ and the autocorrelation function of the reference signal.

The fiber optic coupler 99/1 directed approximately $1 \%$ of light intensity to the reference photodetector. Its signal serves as the reference signal. The probe signal is launched into the sensing fiber through a fiber optic circulator. Light signals reflected from the sensing fiber were received with
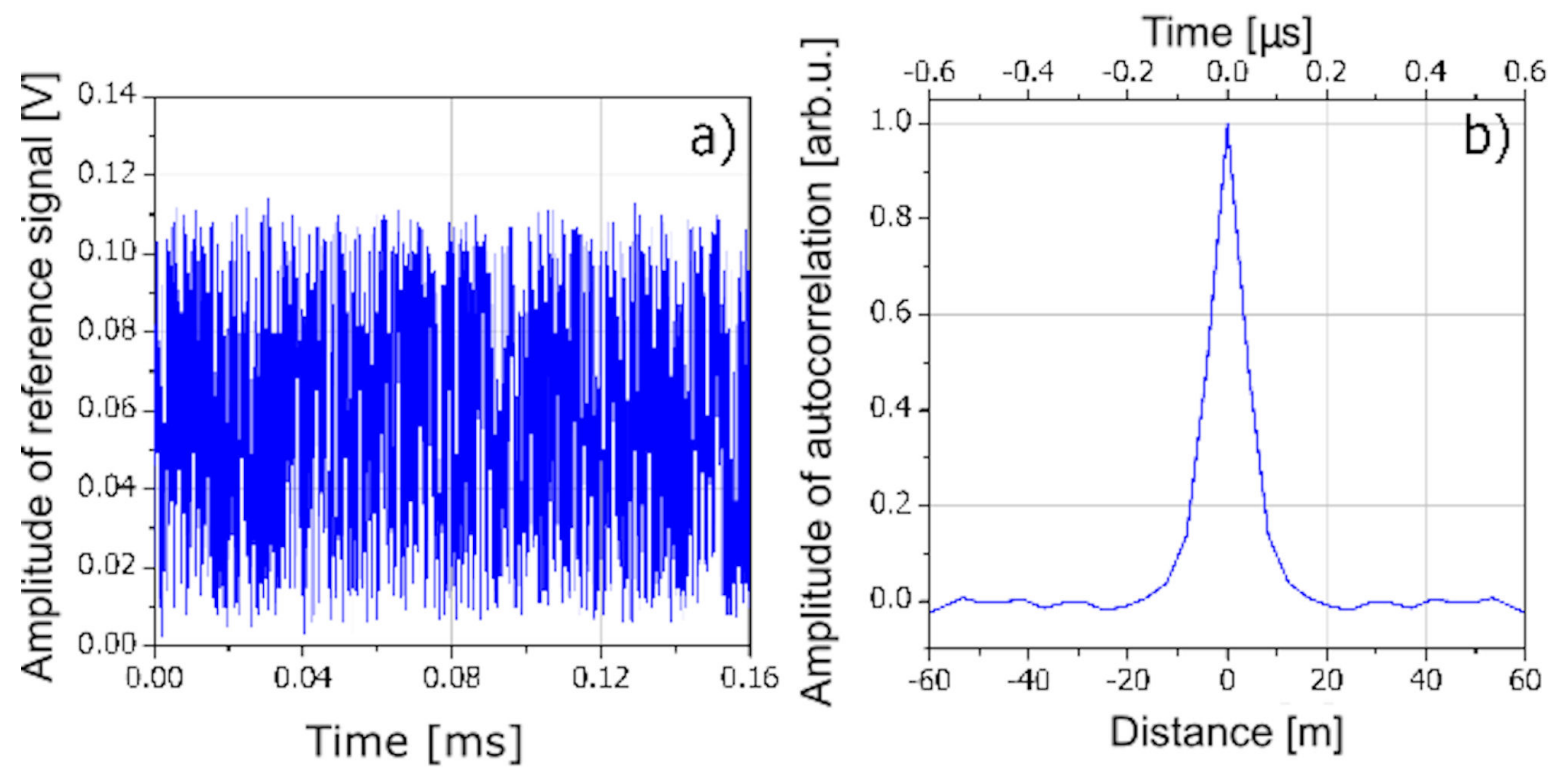

FIGURE 2. a) An example of the reference signal realization, and b) its autocorrelation function. 


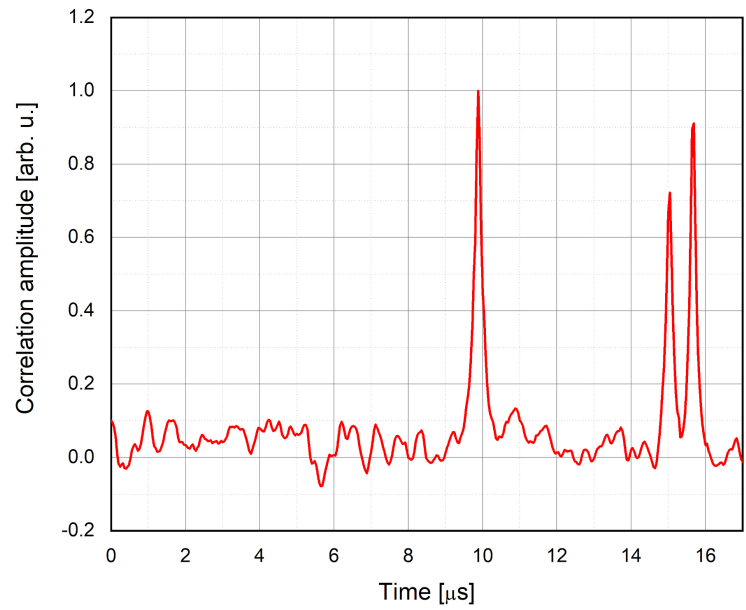

FIGURA 3. Cross-correlation function obtained for optical fiber SMF-28 with 3 FBGs at the fixed wavelength of the laser light. It represents OTDR trace with peaks' positions and amplitudes corresponding to the position and reflectivity of each FBG at the probe light wavelength.

the signal photodetector, which was similar to the reference one. The intensity at the signal photodetector appears as the sum of signals reflected by all FBGs sensors.

Each signal is delayed by the time-of-flight, $t_{d}$, which depends on the position of the particular FBG along the optical fiber, $t_{d}=2 L / v$, where $v$ is the speed of the light in the fiber and $L$ is the distance between the circulator and the particular FBG in the fiber. Signals from the reference and the signal detectors were digitized with a two-channel acquisition board using a sampling rate of $25 \mathrm{MHz}$ and processed in the PC. The multiplexing of the sensor is achieved by calculating the correlation function between a reference and reflected channels. The correlation function appears as a distribution of the reflection coefficient along the optical fiber. In the case of lumped reflectors, such as FBGs are, this distribution contains peaks. The positions and the amplitudes of cross-correlation peaks correspond to positions of FBGs along the optical fiber and to the reflectivity of the correspondent FBG at the wavelength of interrogating light.

FBGs were printed directly into SMF-28 fiber at distances of 1008,1550 , and $1610 \mathrm{~m}$ from the circulator. The length of each grating was $1 \mathrm{~mm}$ and maximum reflectivity of $0.05 \%$. The FBG's reflection spectra were very similar. The mean power of the probe light coupled to the sensing fiber was 1 $\mathrm{mW}$.

A spatial resolution of the correlation-based reflectometers depends mainly on the width of the auto-correlation function of the photo detected signal. For white noise, the autocorrelation function can be approximated by the Delta function, and the spatial resolution for such a probe signal is potentially the best. In practice, the linewidth of the light source or the receiver bandwidth will be the main restrictive factor. In our setup, the laser line bandwidth was about $8 \mathrm{MHz}$ and limited

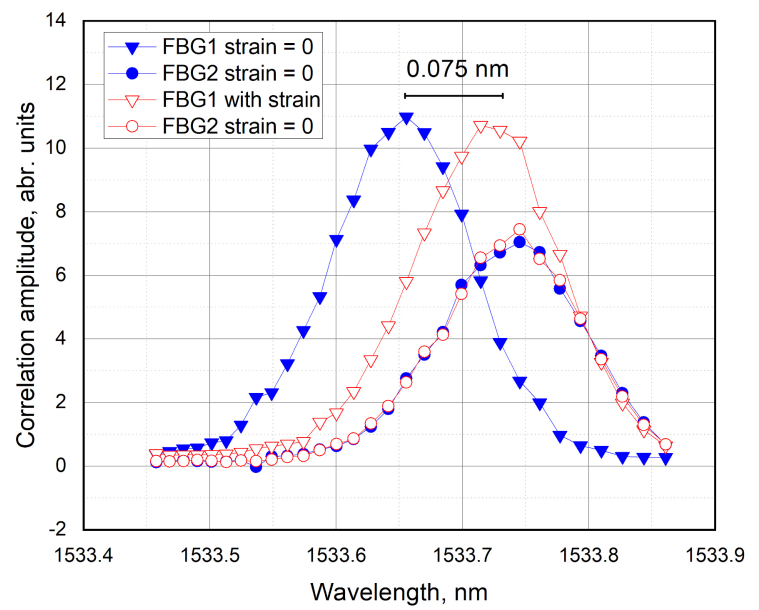

FIGURA 4. Reflection spectra of 2 FBGs measured for 2 levels of applied strain. First measurement was performed without straining the fiber and measured spectra are shown in blue color. Then, axial tension (strain level of $70 \mu$-strains) was applied to one grating with while another one was kept without deformation. Results are shown in red color.

the spatial resolution to about 10 meters. Figure $2 \mathrm{~b}$ ) demonstrates the autocorrelation function of the reference signal shown in Fig. 2a). One can see a good form of the autocorrelation peak with a very low level of side-lobes.

Figure 3 demonstrates the distribution of reflectors along the fiber the measured containing 3 FBGs at distances 1008 , 1550 , and $1610 \mathrm{~m}$ from the circulator, as it is shown in Fig. 1. Measurements were done at a fixed light wavelength. A sample of the cross-correlation function shown in Fig. 3 was obtained after averaging a time of $10 \mathrm{~ms}$. The positions of the three sharp peaks correspond to the spatial positions of the three FBGs along the fiber. The amplitudes of the peaks correspond to the reflectivity of the Bragg gratings at the interrogation wavelength.

As was mentioned above, the reflection spectra of FBGs can be obtained by sweeping the laser light wavelength and measuring amplitudes of the corresponding peaks as a function of wavelength. Figure 4 presents results for two such measurements. Reflection spectra for 2 FBGs were detected firstly without strain applied to any of the gratings. Optical reflection spectra with no strain applied to the fiber Bragg gratings are shown by blue colors. Then, one FBG was strained uniformly while another one was kept without deformation. The longitudinal strain level applied to the grating was $7 \times 10^{-5}$ or $70 \mu \varepsilon$. Results for the second measurements are shown in Fig. 4 with red color. One can see that the Bragg wavelength for the second unstrained FBG remains the same in both measurements, whereas the strained FBG demonstrates so called red shift of $75 \mathrm{pm}$. This value corresponds very well to a typical sensitivity of FBG sensors to strain at a wavelength around $1550 \mathrm{~nm}$. 


\section{Conclusion}

We presented a very simple technique for multiplexing and interrogation of Fiber Bragg Grating sensors using a DFB diode laser operating in CW mode. The technique is based on correlation detection of probe signals reflected by the fiber Bragg gratings. Phase noise of the DFB diode laser converted into intensity noise with an imbalanced interferometer was used as a probe signal. Demultiplexing was performed by calculating the cross-correlation function between the probe reference signal and the signal reflected by the fiber. The cross-correlation function provides a value of reflectivity for each fiber Bragg grating at the probe wavelength. Reflection spectra of FBGs can be obtained by tuning the diode laser wavelength with temperature and calculating the correlation function for each probe wavelength. Results of the experimental verification of the technique were presented.

\section{Acknowledgments}

In memory of our dear friend and colleague, Dr. Eugeny Kuzin.
1. S. Pevec and D. Donlagić, Multiparameter fiber-optic sensors: a review, Optical Engineering 58 (2019) 072009. https: //doi.org/10.1117/1.0E.58.7.072009

2. . A. May-Alarcon et al., Sensor laser de fibra optica con una cavidad de $8.6 \mathrm{~km}$ formada por dos rejillas de Bragg usadas como espejos, Rev. Mex. Fis. 48 (2002) 434.

3. L Xia, Y Wu, U Rahubadde, W Li, TDM Interrogation of Identical Weak FBGs Network Based on Delayed Laser Pulses Differential Detection, IEEE Photonics Journal 10 (2018) 6802308. https://doi.org/10.1109/JPHOT.2018. 2844779

4. D. J. F. Cooper, T. Coroy, and P. W. E. Smith, Time-division multiplexing of large serial fiber-optic Bragg grating sensor arrays, Applied Optics 40 (2001) 2643. https://doi.org/ $10.1364 / \mathrm{AO} .40 .002643$

5. Z. Luo, H. Wen, H. Guo, and M. Yang, A time- and wavelengthdivision multiplexing sensor network with ultra-weak fiber bragg gratings, Optics Express 21 (2013) 22799. https:// doi.org/10.1364/OE.21.022799

6. J. Wang et al., Interrogation of a large-capacity densely spaced fiber Bragg grating array using chaos-based incoherent-optical frequency domain reflectometry, Optics Letters 44 (2019) 5202. https://doi.org/10.1364/OL.44.005202
7. M. Nazarathy et al, Realtime long range complementary correlation optical time domain reflectometer, J. Lightwave Technology 7 (1989) 24. https://doi.org/10.1109/50. 17729

8. N. Takeuchi, N. Sugimoto, H. Baba, and K. Sakurai, Random modulation CW lidar, Applied Optics 22 (1983) 1382. https://doi.org/10.1364/AO.22.001382

9. Y. Wang, B. Wang, and A. Wang, Chaotic correlation optical time domain reflectometer utilizing laser diode, IEEE Photonics Technology Letters 20, (2008) 1636. https://doi. org/10.1109/LPT.2008.2002745

10. A. Arias, M. G. Shlyagin, S. V. Miridonov, and R. Martinez Manuel, Phase-sensitive correlation optical time-domain reflectometer using quantum phase noise of laser light, Optics Express 23 (2015) 30347. https: / / doi.org/10.1364/ OE.23.030347

11. J. H. López, O. Esteban, M. G. Shlyagin, R. Martínez-Manuel, Multipoint Refractometer Based on Combined Correlation and Frequency Multiplexing, IEEE Ptotonics Technology Letters 29 (2017) 1479. https://doi.org/10.1109/LPT.2017. 2723464 